\title{
ECOMPARISON OF STRESS INTENSITY FACTOR SOLUTIONS FOR CYLINDERS WITH AXIAL AND CIRCUMFERENTIAL CRACKS
}

\author{
Naoki MIURA* \\ Central Research Institute of Electric \\ Power Industry \\ Iwado-Kita 2-11-1, Komae-shi, Tokyo, Japan \\ Phone: $+81-3-3480-2111$ \\ Fax: +81-3-3430-2410 \\ E-mail: miura@criepi.denken.or.jp \\ Hiroshi SHIBAMOTO \\ Japan Atomic Power Company
}

\author{
Yukio TAKAHASHI \\ Central Research Institute of Electric \\ Power Industry,
}

\begin{abstract}
Numerous stress intensity factor solutions have been proposed so far depending on the objects of evaluation including the variations of structures, cracks, and applied loads. In applying the flaw evaluation methodology to components of nuclear power plants, the use of reliable stress intensity factor solutions is essential. In this study, cracked cylinders were focused on as one of the typical configurations in actual plants. Existing stress intensity factor solutions for cracked cylinders were reviewed, and the accuracy of these solutions was investigated thorough the comparison with each other. Specific solutions were then recommended for cylindrical structures. Approximate expressions were newly derived for axially through-wall cracked cylinder subjected to linear stress distribution and for circumferentially through-wall cracked cylinder subjected to bending to realize simple evaluation of stress intensity factor. Considering that the cylindrical structures are often replaced with flat plates in the evaluation of actual components, the propriety of the replacement was also studied.
\end{abstract}

Keywords: Stress Intensity Factor, Cylinder, Crack, Fracture Mechanics

\section{INTRODUCTION}

Stress intensity factor plays a dominant role in the regime of the linear elastic fracture mechanics, and numerous solutions have been proposed depending on the objects of evaluation including the variations of structures, cracks, and applied loads. In the flaw evaluation or fitness-for-service codes/standards for components in nuclear power plants, consistent systematization of the stress intensity factor solutions is quite essential in predicting crack propagation behavior due to fatigue and/or SCC and the possible occurrence of unstable fracture.

In this study, cracked cylinders were focused on as one of the most common configurations in actual plants. Existing stress intensity factor solutions for cracked cylinders were collected. The solutions were then compared each other and the applicability of the solutions was evaluated. Since the cylindrical structures are often replaced with flat plate in the evaluation of actual components, the adequacy of the replacement was additionally studied.

\section{COMPARISON OF STRESS INTENSITY FACTOR SOLUTIONS}

Cylindrical structures with cracks can be categorized as shown in Figs. 1(a) to (f). In the figures, $R_{i}, R_{m}$, and $t$ are the inner radius, mean radius, and thickness, $a, c$, and (are the crack depth, half crack length, and half crack 
angle, $u$ is the coordinate in cracked section along thickness, $/(u)$ is the stress distribution, and $/ m, / b$, and $/ b g$ are membrane stress, through-wall bending stress, and global bending stress, respectively. Numerous stress intensity factor solutions have been developed based on detailed numerical analyses for these structures [1-20]. Considering the implementation of the solutions to codes/standards, they have to possess the applicability to wide range of structure and crack sizes, applicability to various applied loading, simplicity of evaluation as well as the accuracy.
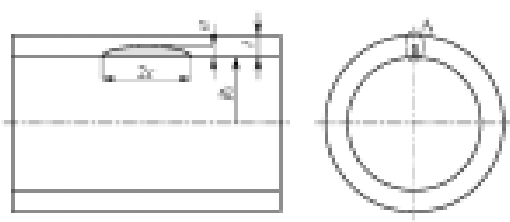

(a) Axially Semi-Elliptical Surface Cracked Cylinder
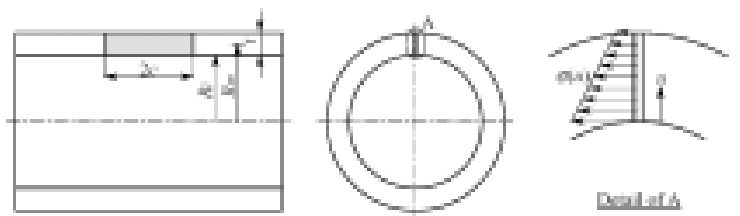

(c) Axially Through-Wall Cracked Cylinder
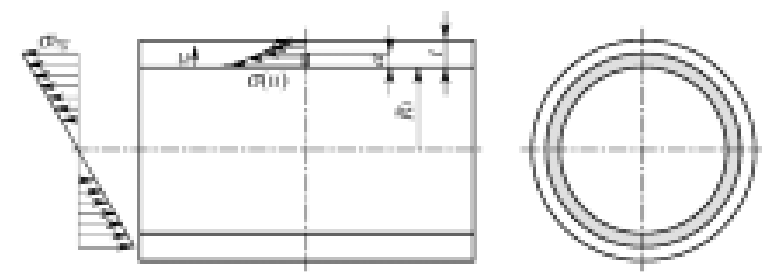

(e) Circumferentially Full-Circumferential Surface Cracked Cylinder

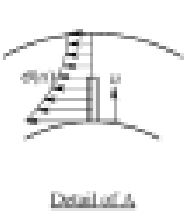

(1)
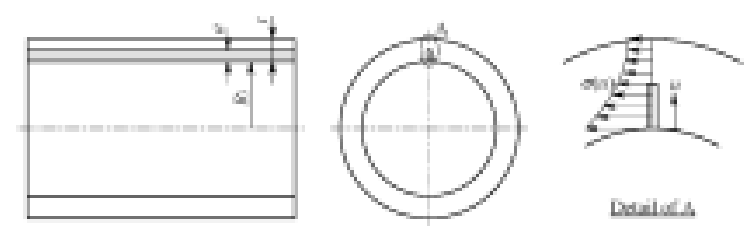

(b) Axially Infinite

Surface Cracked Cylinder
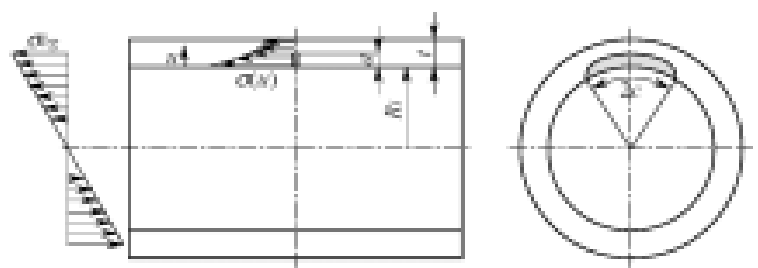
(d) Circumferentially Semi-Elliptical Surface Cracked Cylinder
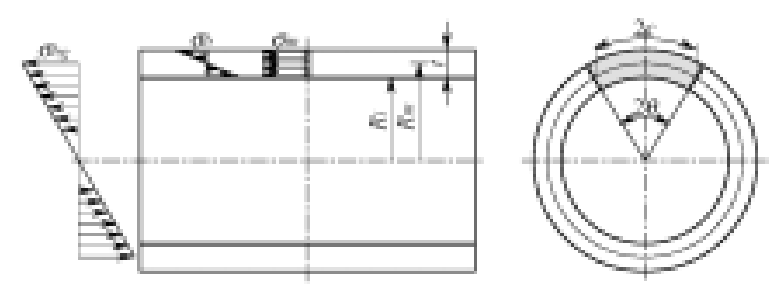

$$
\begin{gathered}
\text { (f) Circumferentially Through-Wall } \\
\text { Cracked Cylinder }
\end{gathered}
$$

Fig. 1 Cylindrical Structures with Cracks

\subsection{Sample Case for Axially Semi-Elliptical Surface Cracked Cylinders}

As an example, available stress intensity factor solutions for axially semi-elliptical surface cracked cylinder shown in Fig. 1(a) are listed in Table 1(a). Figures 2(a) to (d) show the comparison of stress intensity factors obtained from the solutions listed in the table. Here the stress intensity factors are presented by their normalized value defined as $K_{I} / \sigma \sqrt{\pi a}$, and the inner radius-to-thickness ratio, $R_{i} / t$, was fixed to 10 . Not only in the cases illustrated in Figs. 2(a) to (d), but also in the other cases with wide variations of analysis conditions, the solutions were reasonably consistent with each other regardless of crack location (inner/outer surface), evaluation point (deepest/surface point), applied loading (membrane/through-wall bending stress), crack depth-to-thickness ration, $a / t$, and aspect ratio, a/c. This suggests that all of the solutions in Table 1(a) have similar accuracy. From the viewpoint of the applicability to wide range of parameters (crack depth, aspect ratio, and radius-to-thickness ratio), and fineness of matrices, use of CEA's solution [1] could be recommended for general application. 
Table 1 Comparison of Available Stress Intensity Factor Solutions (a) Axially Semi-Elliptical Surface Cracked Cylinder

\begin{tabular}{|c|c|c|c|c|c|}
\hline \multirow{2}{*}{ Solution } & \multirow{2}{*}{ Type of solution } & \multirow{2}{*}{ Applied loading } & \multicolumn{3}{|c|}{ Applicable range } \\
\hline & & & Crack depth & Aspect ratio & $R$ to $t$ ratio \\
\hline $\mathrm{CEA}^{[1]}$ & $\begin{array}{c}\text { Interporation } \\
\text { of tables }\end{array}$ & $\begin{array}{l}\text { 3rd-order polynomial } \\
\text { stress distribution }\end{array}$ & $\begin{array}{c}0.0<a / t \leq 0.8 \\
(6)\end{array}$ & $\begin{array}{c}0.0<a / c \leq 1.0 \\
(6)\end{array}$ & $\begin{array}{c}1 \leq R_{i} / t<\infty \\
(8)\end{array}$ \\
\hline $\mathrm{SAQ}^{[2-4]}$ & $\begin{array}{c}\text { Interporation } \\
\text { of tables }\end{array}$ & $\begin{array}{l}\text { 3rd-order polynomial } \\
\text { stress distribution }\end{array}$ & $\begin{array}{c}0.0<a / t \leq 0.8 \\
(4)\end{array}$ & $\begin{array}{c}0.2 \leq a / c \leq 1.0 \\
(3)\end{array}$ & $\begin{array}{c}4 \leq R_{i} / t \leq 10 \\
(2)\end{array}$ \\
\hline $\mathrm{API}^{[5]}$ & $\begin{array}{c}\text { Interporation } \\
\text { of tables }\end{array}$ & $\begin{array}{l}\text { 4th-order polynomial } \\
\text { stress distribution }\end{array}$ & $\begin{array}{c}0.2 \leq a / t \leq 0.8 \\
(4)\end{array}$ & $\begin{array}{c}1 / 32 \leq a / c \leq 1.0 \\
(6)\end{array}$ & $\begin{array}{c}5 \leq R_{i} / t<\infty \\
(6)\end{array}$ \\
\hline Shiratori ${ }^{[6]}$ & $\begin{array}{c}\text { Interporation } \\
\text { of tables }\end{array}$ & $\begin{array}{l}\text { 3rd-order polynomial } \\
\text { stress distribution }\end{array}$ & $0.1 \leq \underset{(5)}{a / t} \leq 0.8$ & $\begin{array}{c}0.2 \leq a / c \leq 1.0 \\
(4)\end{array}$ & $\begin{array}{c}1 / 9 \leq R_{i} / t \leq 10 \\
(2)\end{array}$ \\
\hline Zahoor $^{[7]}$ & $\begin{array}{l}\text { Approximate } \\
\text { expression }\end{array}$ & Tensile & $0.2 \leq a / t \leq 0.8$ & $1 / 6 \leq a / c \leq 2 / 3$ & $4.5 \leq R_{i} / t \leq 19.5$ \\
\hline
\end{tabular}

( ) denotes number of division for each parameter in table to interpolate correction factor

\section{(b) Axially Infinite Surface Cracked Cylinder}

\begin{tabular}{|c|c|c|c|c|c|}
\hline \multirow{2}{*}{ Solution } & \multirow{2}{*}{ Type of solution } & \multirow{2}{*}{ Applied loading } & \multicolumn{3}{|c|}{ Applicable range } \\
\hline & & & Crack depth & Aspect ratio & $R$ to $t$ ratio \\
\hline $\mathrm{CEA}^{[8]}$ & $\begin{array}{c}\text { Interporation } \\
\text { of tables }\end{array}$ & $\begin{array}{l}\text { 4th-order polynomial } \\
\text { stress distribution }\end{array}$ & $\begin{array}{c}0.0<a / t \leq 0.8 \\
(9)\end{array}$ & $c_{1}$ & $\begin{array}{c}\leq R_{i} / t \leq 400 \\
(9)\end{array}$ \\
\hline $\mathrm{SAQ}^{[2,9]}$ & $\begin{array}{c}\text { Interporation } \\
\text { of tables }\end{array}$ & $\begin{array}{c}\text { Arbitrary } \\
\text { stress distribution } \\
\end{array}$ & $\begin{array}{c}0.0<a / t \leq 0.75 \\
(9)\end{array}$ & - & $\begin{array}{c}0.5 \leq R_{i} / t \leq 4 \\
(4)\end{array}$ \\
\hline $\mathrm{API}^{[5]}$ & $\begin{array}{c}\text { Interporation } \\
\text { of tables }\end{array}$ & $\begin{array}{l}\text { 4th-order polynomial } \\
\text { stress distribution }\end{array}$ & $\begin{array}{c}0.0<a / t \leq 0.8 \\
(5)\end{array}$ & - & $\begin{array}{c}2 \leq R_{i} / t \leq 1000 \\
(9)\end{array}$ \\
\hline Zahoor $^{[7]}$ & $\begin{array}{l}\text { Approximate } \\
\text { expression }\end{array}$ & Tensile & $0.125 \leq a / t \leq 0.75$ & - & $5 \leq R_{i} / t \leq 20$ \\
\hline
\end{tabular}

( ) denotes number of division for each parameter in table to interpolate correction factor

(c) Axially Through-Wall Cracked Cylinder

\begin{tabular}{|c|c|c|c|c|c|}
\hline \multirow{2}{*}{ Solution } & \multirow{2}{*}{ Type of solution } & \multirow{2}{*}{ Applied loading } & \multicolumn{3}{|c|}{ Applicable range } \\
\hline & & & Crack length & Aspect ratio & $R$ to $t$ ratio \\
\hline $\mathrm{CEA}^{[8]}$ & $\begin{array}{c}\text { Interporation } \\
\text { of tables }\end{array}$ & $\begin{array}{c}\text { Linear } \\
\text { stress distribution }\end{array}$ & $0.2 \leq c)_{(25)^{2}} \sqrt{R_{m}} \leq 5.0$ & - & Not provided \\
\hline $\mathrm{SAQ}^{[2,10]}$ & $\begin{array}{c}\text { Interporation } \\
\text { of tables }\end{array}$ & $\begin{array}{c}\text { Tensile, } \\
\text { Through-wall bending }\end{array}$ & \begin{tabular}{|c|}
$0.0<c / t \leq 12.5$ \\
$(9)$
\end{tabular} & - & $\begin{array}{c}10 \leq R_{i} / t \leq 20 \\
(2)\end{array}$ \\
\hline $\operatorname{API}^{[5,11,12]}$ & $\begin{array}{c}\text { Interporation } \\
\text { of tables }\end{array}$ & $\begin{array}{c}\text { Tensile, } \\
\text { Through-wall bending }\end{array}$ & $0.0<c / \sqrt{R_{i} t} \leq 6.88$ & - & $\begin{array}{c}3 \leq R_{i} / t \leq 100 \\
(6)\end{array}$ \\
\hline $\operatorname{ASME}^{[13]}$ & $\begin{array}{l}\text { Approximate } \\
\text { expression }\end{array}$ & Tensile & $0.0<c / \sqrt{R_{m} t} \leq 5.0$ & - & Not provided \\
\hline $\mathrm{AEA}^{[14]}$ & $\begin{array}{c}\text { Interporation } \\
\text { of tables }\end{array}$ & $\begin{array}{c}\text { Tensile, } \\
\text { Through-wall bending }\end{array}$ & $0.0<c / \sqrt{R_{m} t} \leq 6.72$ & - & $\begin{array}{c}2.5 \leq R_{i} / t \leq 99.5 \\
(6)\end{array}$ \\
\hline
\end{tabular}

( ) denotes number of division for each parameter in table to interpolate correction factor 
Table 1 Comparison of Available Stress Intensity Factor Solutions (Cont'd) (d) Circumferentially Semi-Elliptical Surface Cracked Cylinder

\begin{tabular}{|c|c|c|c|c|c|}
\hline \multirow{2}{*}{ Solution } & \multirow{2}{*}{ Type of solution } & \multirow{2}{*}{ Applied loading } & \multicolumn{3}{|c|}{ Applicable range } \\
\hline & & & Crack depth & Aspect ratio & $R$ to $t$ ratio \\
\hline $\mathrm{CEA}^{[1,15]}$ & $\begin{array}{c}\text { Interporation } \\
\text { of tables }\end{array}$ & $\begin{array}{l}\text { 3rd-order polynomial } \\
\text { stress distribution, } \\
\text { Global bending } \\
\end{array}$ & $\begin{array}{c}0.0<a / t \leq 0.8 \\
\text { (6) }\end{array}$ & $\begin{array}{c}0.0<a / c \leq 1.0 \\
\text { (6) }\end{array}$ & $\begin{array}{c}1 \leq R_{i} / t<\infty \\
\text { (8) }\end{array}$ \\
\hline $\mathrm{SAQ}^{[2,3,16]}$ & $\begin{array}{c}\text { Interporation } \\
\text { of tables }\end{array}$ & $\begin{array}{c}\text { 3rd-order polynomial } \\
\text { stress distribution, } \\
\text { Global bending }\end{array}$ & $\begin{array}{c}0.0<a / t \leq 0.8 \\
\text { (5) }\end{array}$ & $\begin{array}{c}1 / 16 \leq a / c \leq 1.0 \\
(5)\end{array}$ & $\begin{array}{c}5 \leq R_{i} / t \leq 10 \\
\text { (2) }\end{array}$ \\
\hline $\mathrm{API}^{[5]}$ & $\begin{array}{c}\text { Interporation } \\
\text { of tables }\end{array}$ & $\begin{array}{l}\text { 4th-order polynomial } \\
\text { stress distribution }\end{array}$ & $\begin{array}{c}0.2 \leq a / t \leq 0.8 \\
(4)\end{array}$ & $\begin{array}{c}1 / 32 \leq a / c \leq 1.0 \\
(6)\end{array}$ & $\begin{array}{c}5 \leq R_{i} / t<\infty \\
(6)\end{array}$ \\
\hline Shiratori $^{[6]}$ & $\begin{array}{c}\text { Interporation } \\
\text { of tables }\end{array}$ & Tensile, Global bending & $\begin{array}{c}0.1 \leq a / t \leq 0.8 \\
(5)\end{array}$ & $\begin{array}{c}0.2 \leq a / c \leq 1.0 \\
(4)\end{array}$ & $\begin{array}{c}1.25 \leq R_{i} / t \leq 10 \\
(5)\end{array}$ \\
\hline Zahoor $^{[7]}$ & $\begin{array}{l}\text { Approximate } \\
\text { expression }\end{array}$ & Tensile & $0.2 \leq a / t \leq 0.8$ & $1 / 6 \leq a / c \leq 2 / 3$ & $4.5 \leq R_{i} / t \leq 19.5$ \\
\hline
\end{tabular}

( ) denotes number of division for each parameter in table to interpolate correction factor

(e) Circumferentially Full-Circumferential Surface Cracked Cylinder

\begin{tabular}{|c|c|c|c|c|c|}
\hline \multirow{2}{*}{ Solution } & \multirow{2}{*}{ Type of solution } & \multirow{2}{*}{ Applied loading } & \multicolumn{3}{|c|}{ Applicable range } \\
\hline & & & Crack depth & Aspect ratio & $R$ to $t$ ratio \\
\hline $\mathrm{CEA}^{[8]}$ & $\begin{array}{l}\text { Interporation } \\
\text { of tables }\end{array}$ & $\begin{array}{l}\text { 4th-order polynomial } \\
\text { stress distribution, } \\
\text { Global bending }\end{array}$ & $\begin{array}{c}0.0<a / t \leq 0.8 \\
\text { (9) }\end{array}$ & - & $\begin{array}{c}1 \leq R_{i} / t \leq 400 \\
\text { (9) }\end{array}$ \\
\hline $\mathrm{SAQ}^{[2,9]}$ & $\begin{array}{c}\text { Interporation } \\
\text { of tables }\end{array}$ & $\begin{array}{l}\text { 3rd-order polynomial } \\
\text { stress distribution }\end{array}$ & $\begin{array}{c}0.0<a / t \leq 0.6 \\
(7)\end{array}$ & - & $\begin{array}{c}7 / 3 \leq R_{i} / t \leq 10 \\
(3)\end{array}$ \\
\hline $\mathrm{API}^{[5]}$ & $\begin{array}{c}\text { Interporation } \\
\text { of tables }\end{array}$ & $\begin{array}{l}\text { 4th-order polynomial } \\
\text { stress distribution }\end{array}$ & $\begin{array}{c}0.0<a / t \leq 0.8 \\
(5)\end{array}$ & - & $\begin{array}{c}2 \leq R_{i} / t \leq 1000 \\
(9)\end{array}$ \\
\hline Zahoor $^{[7]}$ & $\begin{array}{l}\text { Approximate } \\
\text { expression }\end{array}$ & Tensile & $0.125 \leq a / t \leq 0.75$ & - & $5 \leq R_{i} / t \leq 20$ \\
\hline
\end{tabular}

( ) denotes number of division for each parameter in table to interpolate correction factor

\section{(f) Circumferentially Through-Wall Cracked Cylinder}

\begin{tabular}{|c|c|c|c|c|c|}
\hline \multirow{2}{*}{ Solution } & \multirow{2}{*}{ Type of solution } & \multirow{2}{*}{ Applied loading } & \multicolumn{3}{|c|}{ Applicable range } \\
\hline & & & Crack angle & Aspect ratio & $R$ to $t$ ratio \\
\hline $\mathrm{CEA}^{[8,17]}$ & $\begin{array}{c}\text { Interporation } \\
\text { of tables }\end{array}$ & Tensile, Global bending & $\begin{array}{c}0^{\circ}<\theta \leq 110^{\circ} \\
(12)\end{array}$ & - & $\begin{array}{c}1 \leq R_{i} / t \leq 80 \\
(7)\end{array}$ \\
\hline $\mathrm{SAQ}^{[2,8,18]}$ & $\begin{array}{l}\text { Interporation } \\
\text { of tables }\end{array}$ & $\begin{array}{c}\text { Tensile, } \\
\text { Through-wall bending, } \\
\text { Global bending }\end{array}$ & $\begin{array}{c}0^{\circ}<\theta \leq 90^{\circ} \\
(11)\end{array}$ & - & $\begin{array}{c}5 \leq R_{i} / t \leq 20 \\
\text { (4) }\end{array}$ \\
\hline $\operatorname{API}^{[5,11,12]}$ & $\begin{array}{l}\text { Interporation } \\
\text { of tables }\end{array}$ & $\begin{array}{l}\text { Tensile, } \\
\text { Through-wall bending }\end{array}$ & $\begin{array}{c}\text { Depend on } R_{i} / t \\
0^{\circ}<\theta \leq 82^{\circ} \\
\text { when } R_{i} / t=10 \\
\end{array}$ & - & $\begin{array}{c}3 \leq R_{i} / t \leq 100 \\
(6)\end{array}$ \\
\hline Zahoor $^{[7]}$ & $\begin{array}{l}\text { Approximate } \\
\text { expression }\end{array}$ & Tensile, Global bending & $0^{\circ}<\theta \leq 99^{\circ}$ & - & $4.5 \leq R_{i} / t \leq 19.5$ \\
\hline Klecker $^{[19]}$ & $\begin{array}{l}\text { Approximate } \\
\text { expression }\end{array}$ & Tensile, Global bending & $0^{\circ}<\theta \leq 110^{\circ}$ & - & $4.5 \leq R_{i} / t \leq 14.5$ \\
\hline Takahashi $^{[20]}$ & $\begin{array}{l}\text { Approximate } \\
\text { expression }\end{array}$ & Tensile, Global bending & $0^{\circ}<\theta \leq 110^{\circ}$ & - & $1 \leq R_{i} / t \leq 80$ \\
\hline $\mathrm{AEA}^{[14]}$ & $\begin{array}{l}\text { Interporation } \\
\text { of tables }\end{array}$ & $\begin{array}{c}\text { Tensile, } \\
\text { Through-wall bending, } \\
\text { Global bending }\end{array}$ & $0.0<a / \sqrt{R_{m} t} \leq 6.72$ & - & $\begin{array}{c}2.5 \leq R_{i} / t \leq 99.5 \\
(6)\end{array}$ \\
\hline
\end{tabular}

( ) denotes number of division for each parameter in table to interpolate correction factor 


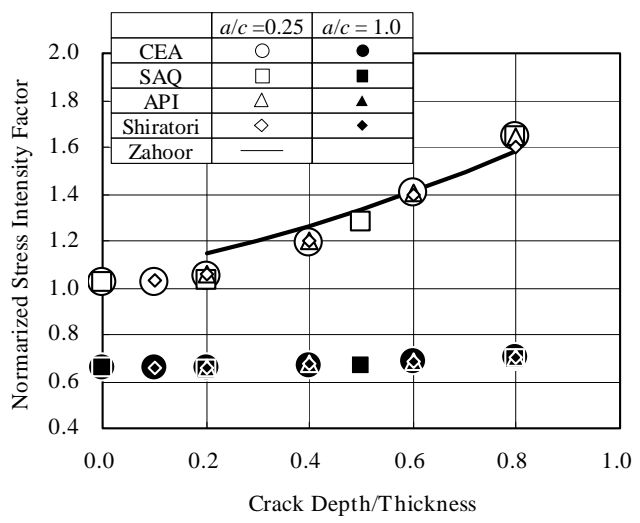

(a) Inner Surface Crack, Deepest Point, Membrane Stress

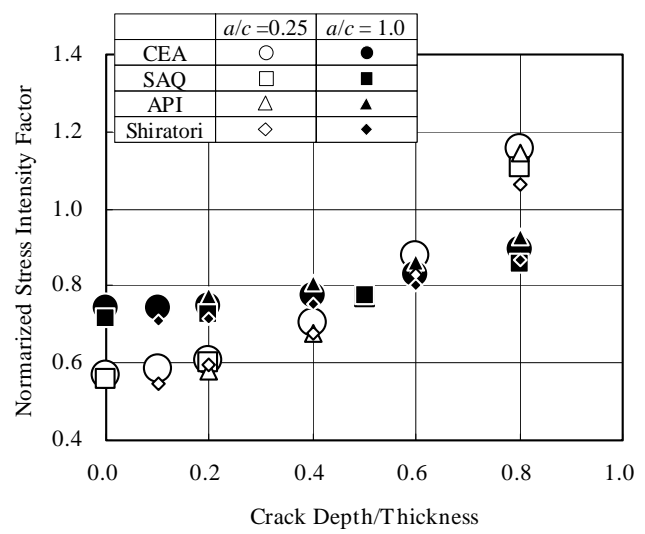

(c) Inner Surface Crack, Surface Point, Membrane Stress

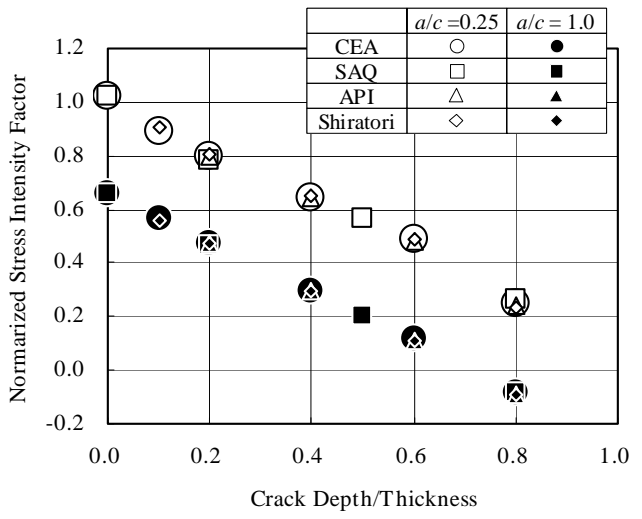

(b) Inner Surface Crack, Deepest Point, Through-Wall Bending Stress

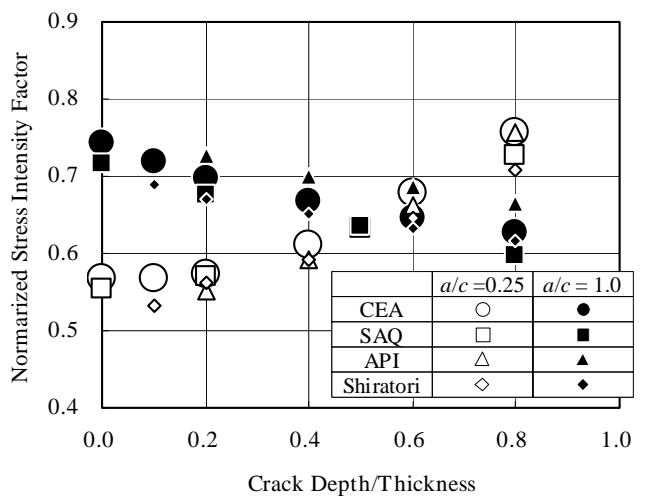

(d) Inner Surface Crack, Surface Point, Through-Wall Bending Stress

Fig. 2 Comparison of Stress Intensity Factors for Axially Semi-Elliptical Surface Cracked Cylinder $\left(R_{i} / t=10\right)$

\subsection{Sample Case for Axially Infinite Surface Cracked Cylinders}

As another example, stress intensity factor solutions for axially infinite surface cracked cylinder shown in Fig. 1(b) are listed in Table 1(b). Figures 3(a) and (b) show the comparison of stress intensity factors obtained from these solutions. The inner radius-to-thickness ratio, $R_{i} / t$, was fixed to 10 again. All of the cases with various analysis conditions including the cases in Figs. 3(a) and (b) again show good consistencies with each other regardless of the differences of crack location (inner/outer surface), applied loading (membrane/through-wall bending stress), and crack depth-to-thickness ratio. This suggests that all of the solutions in Table 1(b) have similar accuracy. From the viewpoint of the applicability to wide range of parameters, use of CEA's solution [8] can be recommended.

\subsection{Comparison for Other Cylindrical Structures}

The similar review and comparison were conducted for the other types of cracks shown in Figs. 1(c) to (f). Available stress intensity factor solutions for these cracks are listed in Tables 1(c) to (f), respectively. Differences between the solutions were eventually similar to those shown above. 


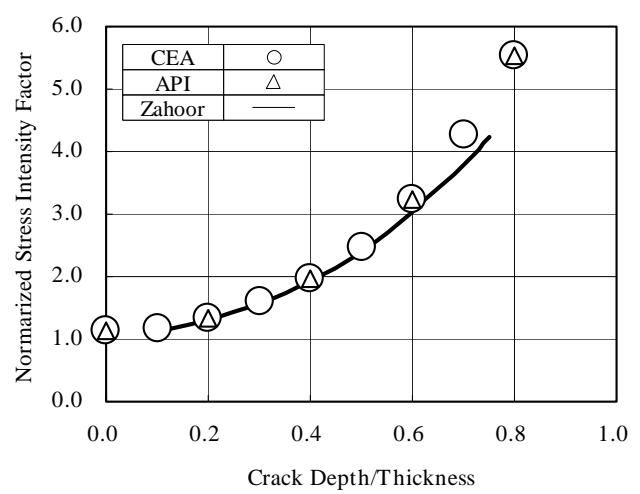

(a) Inner Surface Crack, Membrane Stress

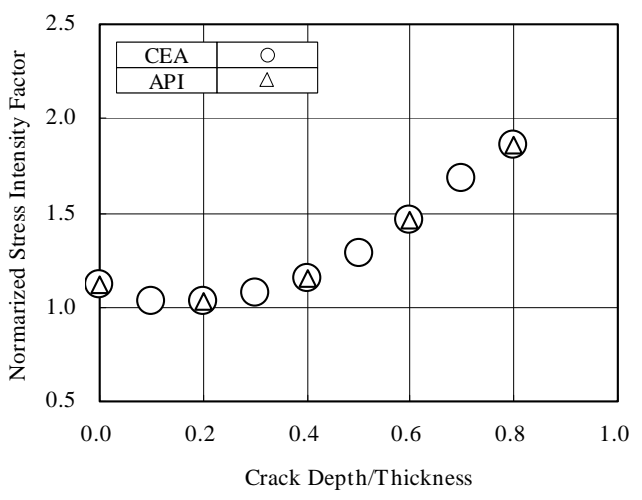

(b) Inner Surface Crack, Through-Wall Bending Stress

Fig. 3 Comparison of Stress Intensity Factors for Axially Infinite Surface Cracked Cylinder $\left(R_{i} / t=10\right)$

\section{DEVELOPMENT OF APPROXIMATE EXPRESSIONS OF STRESS INTENSITY FACTOR}

In most of the solutions reviewed in the preceding section, correction factors are given as discrete values in tables and need to be interpolated in regard to geometrical parameters such as $a / t, a / c$, and $R_{i} / t$. Although linear interpolation in terms of these parameters is usually employed, its effectiveness is not trivial and interpolation by nonlinear functions might be more proper in some cases. In addition, expressions are much easier to implement than matrices storing many components. In these regards, approximate expressions with similar accuracy and applicable range would be useful. In this section, approximate expressions for through-wall cracks were derived.

\subsection{Axially Through-Wall Crack Subjected to Linear Stress Distribution}

Based on the comparison of the solutions shown in Table 1(c), CEA's solution [1] was selected as the base of the solution for an axially through-wall cracked cylinder subjected to linear stress distribution along thickness.

$$
\begin{aligned}
& K_{I, \text { outer }}=\left[F_{0, \text { outer }} \sigma_{0}+F_{1, \text { outer }} \sigma_{1}\right] \sqrt{\pi c}, \quad K_{I, \text { inner }}=\left[F_{0, \text { inner }} \sigma_{0}+F_{1, \text { inner }} \sigma_{1}\right] \sqrt{\pi c} \\
& \sigma(u)=\sigma_{0}+\sigma_{1} \frac{u}{t}
\end{aligned}
$$

where subscriptions "outer" and "inner" denote the outer and inner surface points of the crack front, respectively. The correction factors, $F_{0, \text { outer }}, F_{1, \text { outer }}, F_{0, \text { inner }}$, and $F_{1, \text { inner }}$, are given in tabulated forms in Reference [1]. Since these factors are the function of only $\lambda=c / \sqrt{R_{m} t}$, they could be approximated by polynomial equations in regard to $\left\{. \quad F_{0, \text { outer }}, F_{0, \text { inner }}\right.$, and $F_{1, \text { inner }}$ were set to 1 at $\left\{=0\right.$, and $F_{1, \text { outer }}$ was set to 0 at $\{=0$ so that the factors were asymptotic to theoretical solutions, thus the following expressions were derived by the least square method.

$$
\begin{aligned}
& F_{0, \text { outer }}=F_{0, \text { inner }}=1+0.1847 \lambda+0.4427 \lambda^{2}-0.1112 \lambda^{3}+0.0095 \lambda^{4} \\
& F_{1, \text { outer }}=0.0492 \lambda+0.359 \lambda^{2}-0.132 \lambda^{3}+0.0219 \lambda^{4}-0.0014 \lambda^{5} \\
& F_{1, \text { inner }}=1+0.0643 \lambda+0.2116 \lambda^{2}-0.0531 \lambda^{3}+0.0046 \lambda^{4}
\end{aligned}
$$

Figure 4 shows the comparison of the correction factors from CEA's solution [1] and those from the approximate expressions. Satisfactory agreement was achieved over the whole range of $\{(0.2$ to 5$)$ with the error less than $6.2 \%$. 


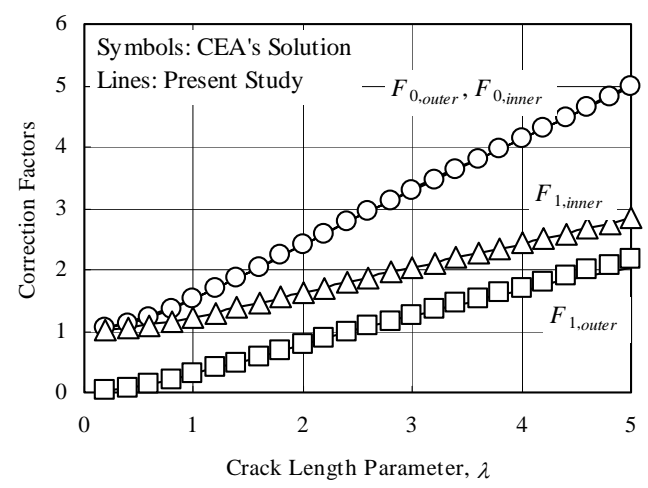

Fig. 4 Comparison of Correction Factors for Axially Through-Wall Cracked Cylinder Subjected to Linear Stress Distribution

\subsection{Circumferentially Through-Wall Crack Subjected to Through-Wall Bending}

For a circumferentially through-wall cracked cylinder, Takahashi's solution [20] is available in the form of expressions for tensile and global bending. On the other hand, SAQ's tabulated solution [2] is a unique solution currently available for through-wall bending, and no approximate expressions were provided so far. The correction factors in SAQ's solution are function of $\left(U \square\right.$ and $R_{i} / t$. They were converted to the same manner as the form of the Takahashi's solution, then the approximate expressions were derived by the least square method as follows:

$$
\begin{gathered}
K_{I, \text { outrer }}=F_{b, \text { outer }} \sigma_{b} \sqrt{\pi c} \\
F_{b, \text { outer }}=A_{b, \text { outer }}+B_{b, \text { outer }} \frac{\theta}{\pi}+C_{b, \text { outer }}\left(\frac{\theta}{\pi}\right)^{2}+D_{b, \text { outer }}\left(\frac{\theta}{\pi}\right)^{3}+E_{b, \text { outer }}\left(\frac{\theta}{\pi}\right)^{4} \\
{\left[\begin{array}{l}
A_{b, \text { outer }} \\
B_{b, \text { outer }} \\
C_{b, \text { outer }} \\
D_{b, \text { outer }} \\
E_{b, \text { outer }}
\end{array}\right]=\left[\begin{array}{ccc}
-1.112 & -0.3412 & -0.09810 \\
5.179 & 2.506 & 2.000 \\
-24.09 & -23.17 & -15.40 \\
54.06 & 63.92 & 38.38 \\
-44.57 & -55.92 & -31.25
\end{array}\right]\left[\begin{array}{c}
1 \\
\xi \\
\xi^{2}
\end{array}\right]} \\
K_{I, \text { inner }}=F_{b, \text { inner }} \sigma_{b} \sqrt{\pi c} \\
F_{b, \text { inner }}=A_{b, \text { inner }}+B_{b, \text { inner }} \frac{\theta}{\pi}+C_{b, \text { inner }}\left(\frac{\theta}{\pi}\right)^{2}+D_{b, \text { inner }}\left(\frac{\theta}{\pi}\right)^{3}+E_{b, \text { inner }}\left(\frac{\theta}{\pi}\right)^{4} \\
{\left[\begin{array}{l}
A_{b, \text { inner }} \\
B_{b, \text { inner }} \\
C_{b, \text { inner }} \\
D_{b, \text { inner }} \\
E_{b, \text { inner }}
\end{array}\right]=\left[\begin{array}{ccc}
1.132 & 0.4708 & 0.1453 \\
-8.548 & -7.285 & -4.826 \\
44.72 & 61.79 & 40.04 \\
-115.6 & -191.5 & -117.1 \\
103.5 & 185.0 & 109.0
\end{array}\right]\left[\begin{array}{l}
1 \\
\xi \\
\xi^{2}
\end{array}\right]} \\
\xi=\log \left(t / R_{m}\right)
\end{gathered}
$$

Figure 5 shows the comparison of the correction factors from SAQ's solution [2] and those from the approximate expressions. Good agreement can be seen over the whole applicable range $\left(5<R_{i} / t<20,0<(/ \square<\right.$ $0.5)$. The error was at most $6.0 \%$.

Based on the comparison of stress intensity factor solutions in the preceding section and the development of approximate expressions in this section, the recommended solutions for specific cracked cylinders are summarized as shown in Table 2. 


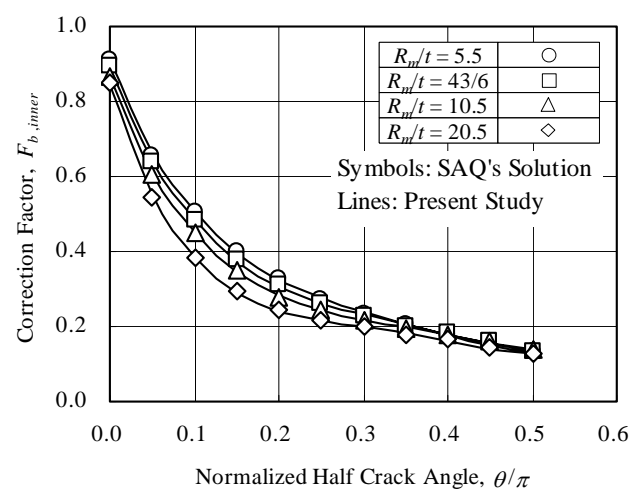

(a) Inner Surface Point

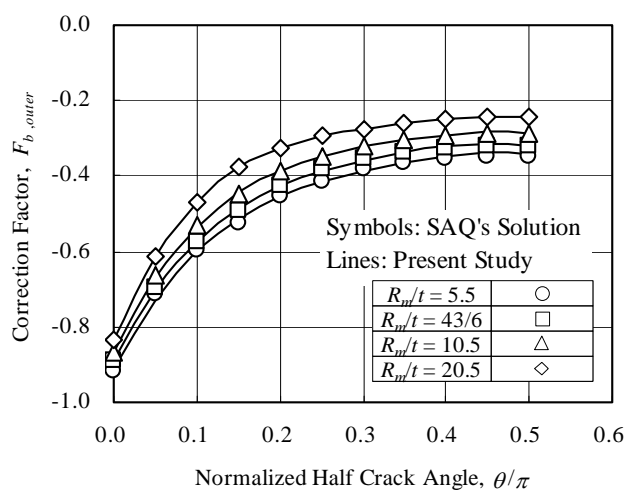

(b) Outer Surface Point

Fig. 5 Comparison of Correction Factors for Circumferential Through-Wall Cracked Cylinder Subjected to Through-Wall Bending

Table 2 Summary of Recommended Solutions for Cracked Cylinders

\begin{tabular}{|c|c|c|c|c|c|c|c|}
\hline \multicolumn{2}{|c|}{ Characteristic of crack } & \multicolumn{6}{|c|}{ Recommended solution } \\
\hline \multirow{2}{*}{ Orientation } & \multirow{2}{*}{ Type } & \multirow{2}{*}{ Solution } & \multirow{2}{*}{ Type of solution } & \multirow{2}{*}{ Applied loading } & \multicolumn{3}{|c|}{ Applicable range } \\
\hline & & & & & Crack depth & Aspect ratio & $R$ to $t$ ratio \\
\hline \multirow{3}{*}{ Axial } & $\begin{array}{l}\text { Semi-elliptical } \\
\text { surface }\end{array}$ & $\mathrm{CEA}^{[1]}$ & $\begin{array}{c}\text { Interporation } \\
\text { of tables }\end{array}$ & $\begin{array}{l}\text { 3rd-order polynomial } \\
\text { stress distribution }\end{array}$ & $0.0<a / t \leq 0.8$ & $0.0<a / c \leq 1.0$ & $1 \leq R_{i} / t<\infty$ \\
\hline & $\begin{array}{l}\text { Infinite } \\
\text { surface }\end{array}$ & $\mathrm{CEA}^{[8]}$ & $\begin{array}{c}\text { Interporation } \\
\text { of tables }\end{array}$ & $\begin{array}{l}\text { 4th-order polynomial } \\
\text { stress distribution }\end{array}$ & $0.0<a / t \leq 0.8$ & - & $1 \leq R_{i} / t \leq 400$ \\
\hline & Through-wall & $\begin{array}{l}\text { Present } \\
\text { Study } \\
\end{array}$ & $\begin{array}{l}\text { Approximate } \\
\text { expression }\end{array}$ & Linear stress distribution & $\begin{array}{c}\text { (Crack length) } \\
0.2 \leq c / \sqrt{R_{m} t} \leq 5.0 \\
\end{array}$ & - & Not provided \\
\hline \multirow{4}{*}{ Circumferential } & $\begin{array}{l}\text { Semi-elliptical } \\
\text { surface }\end{array}$ & $\mathrm{CEA}^{[1,15]}$ & $\begin{array}{c}\text { Interporation } \\
\text { of tables }\end{array}$ & $\begin{array}{l}\text { 3rd-order polynomial } \\
\text { stress distribution, } \\
\text { Global bending }\end{array}$ & $0.0<a / t \leq 0.8$ & $0.0<a / c \leq 1.0$ & $1 \leq R_{i} / t<\infty$ \\
\hline & $\begin{array}{c}\text { Full } \\
\text { circumferential } \\
\text { surface }\end{array}$ & $\mathrm{CEA}^{[8]}$ & $\begin{array}{c}\text { Interporation } \\
\text { of tables }\end{array}$ & $\begin{array}{l}\text { 3rd-order polynomial } \\
\text { stress distribution, } \\
\text { Global bending }\end{array}$ & $0.0<a / t \leq 0.8$ & - & $1 \leq R_{i} / t \leq 400$ \\
\hline & \multirow{2}{*}{ Through-wall } & Takahashi $^{[20]}$ & $\begin{array}{c}\text { Approximate } \\
\text { expression }\end{array}$ & Tensile, Global bending & $\begin{array}{c}\text { (Crack angle) } \\
0^{\circ}<\theta \leq 110^{\circ} \\
\end{array}$ & - & $1 \leq R_{i} / t \leq 80$ \\
\hline & & $\begin{array}{l}\text { Present } \\
\text { Study }\end{array}$ & $\begin{array}{l}\text { Approximate } \\
\text { expression }\end{array}$ & Through-wall bending & $\begin{array}{c}\text { (Crack angle) } \\
0^{\circ}<\theta \leq 90^{\circ} \\
\end{array}$ & - & $5 \leq R_{i} / t \leq 20$ \\
\hline
\end{tabular}

$a$ : Crack depth, $c$ : Half crack length, $R_{i}$ : Inner radius, $R_{m}$ : Mean radius, $t$ : Thickness, $\theta$ : Half crack angle

\section{COMPARISON OF CRACKED CYLINDER SOLUTIONS WITH CRACKED PLATE SOLUTIONS}

When stress intensity factors are evaluated for actual components with postulated cracks, axisymmetric structures are often replaced with cylindrical structures. In addition, the stress intensity factor solutions for cracked plates are sometimes substituted for those for cracked cylinders. The following replacement can be generally considered concerning surface cracked structures:

$>$ Replacement of axially semi-elliptical surface cracked cylinders with semi-elliptical surface cracked plates,

$>$ Replacement of axially infinite surface cracked cylinders with single-edge cracked plates,

$>$ Replacement of circumferentially semi-elliptical surface cracked cylinders with semi-elliptical surface cracked plates, and

$>$ Replacement of circumferentially full-circumferential surface cracked cylinders with single-edge cracked plates.

The adequacy of the replacement was studied in this section. The recommended stress intensity factor solutions shown in Table 1 were used for cracked cylinders, and Raju-Newman's solution [21] and Tada's solution [22] were adopted for semi-elliptical surface cracked plates and single-edge cracked plates, respectively. 


\subsection{Sample Case for Axially Semi-Elliptical Surface Crack}

Figures 6(a) to (d) show examples evaluating the effect of radius-to-thickness ratio on stress intensity factors for axially semi-elliptical surface cracked cylinders. The reciprocal of $R_{i} / t$ is taken as the horizontal axis and the normalized stress intensity factor defined as $K_{I} / \sigma \sqrt{\pi a}$ is taken as the vertical axis. The normalized stress intensity factors for the equivalent semi-elliptical surface cracked plates were also plotted on the figure, where the width of the plate was assumed to be infinity. As the cylinder becomes thinner $\left(t / R_{i}\right.$ is smaller), the normalized stress intensity factors for inner and outer cracks get closer to each other, and they eventually converge to the same value at $t / R_{i}=0$, which is almost equal to the normalized stress intensity factor for the plate, in spite of the differences of crack location (inner/outer surface), evaluation point (deepest/surface point), and applied loading (membrane/through-wall bending stress).

Variations of the normalized stress intensity factors with normalized crack depth are compared in Figs. 7(a) to (d). For the deepest points of deep cracks (e.g. $a / t=0.8)$ at outer surface in the thick cylinder $\left(R_{i} / t=10\right)$, the normalized stress intensity factors are slightly higher than those for cracked plates, however, the normalized stress intensity factors for cracked cylinders are in good agreement with those for the cracked plates in all of the other cases. For the cracks with alt $\delta$ 0.6, the errors of the stress intensity factor at the deepest points due to the replacement of the cracked cylinders with the cracked plates are less than $7 \%, 9 \%$, and $12 \%$ for $R_{i} / t=\square, 80$, and 10 , respectively. The errors at the surface points due to the replacement are less than $6 \%, 6 \%$, and $8 \%$ for $R_{i} / t=\square, 80$, and 10, respectively. Thus we can conclude that the replacement of axially semi-elliptical surface cracked cylinders with semi-elliptical surface cracked plates is acceptable in the case of alt $\delta 0.6$ and $R_{i} / t \varepsilon 10$.

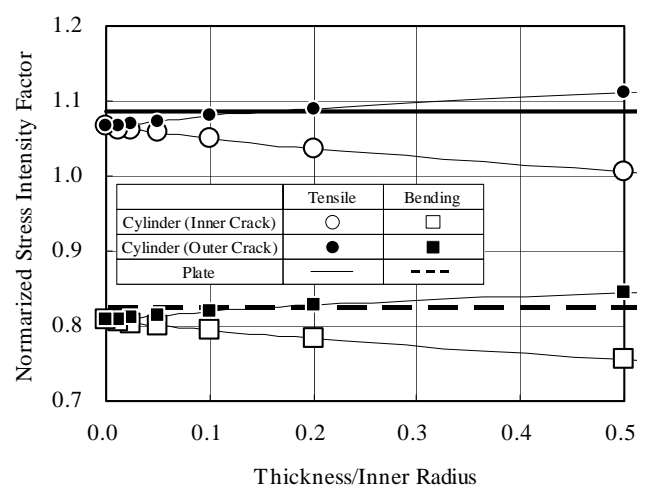

(a) Deepest Point, $a / c=0.25, a / t=0.2$

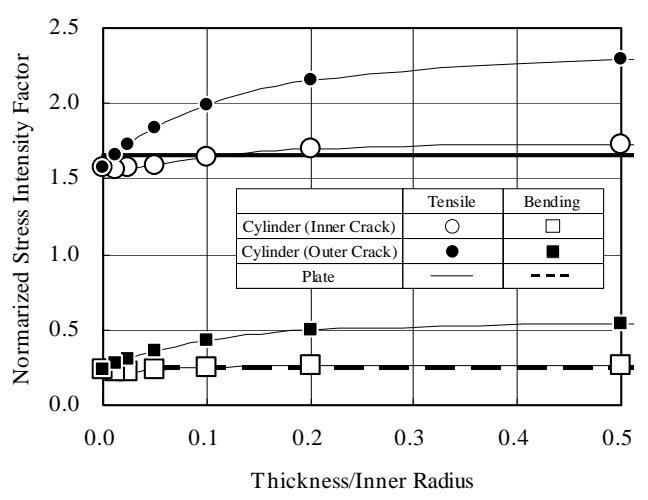

(c) Deepest Point, $a / c=0.25, a / t=0.8$

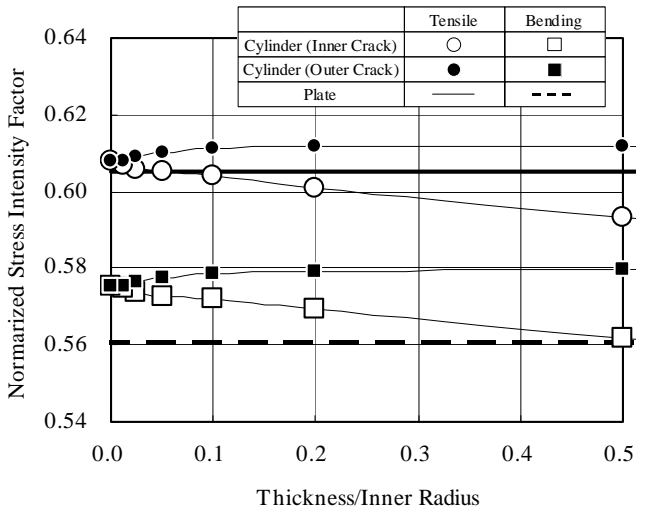

(b) Surface Point, $a / c=0.25, a / t=0.2$

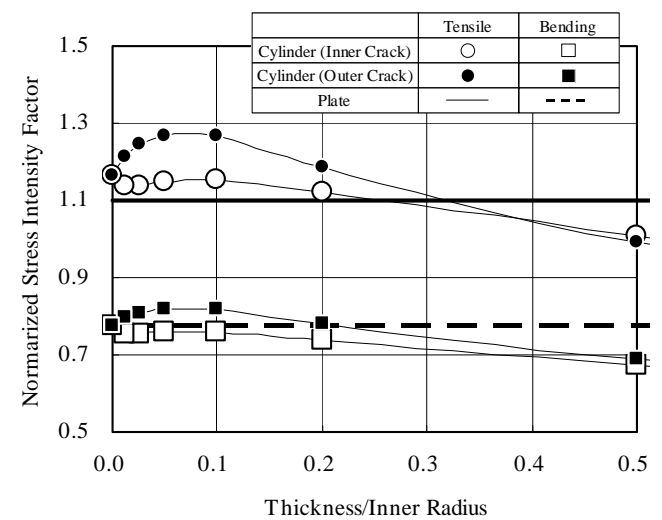

(d) Surface Point, $a / c=0.25, a / t=0.8$

Fig. 6 Effect of Radius-to-Thickness Ratio on Stress Intensity Factors for Axially Semi-Elliptical Surface Cracked Cylinders 


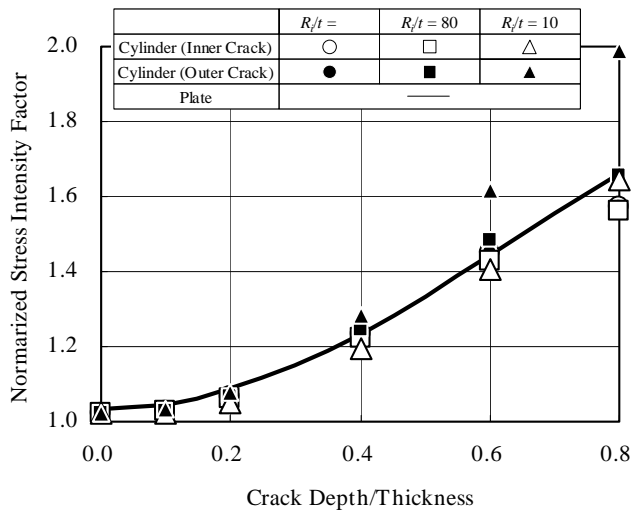

(a) Membrane Stress, Deepest Point, $a / c=0.25$,

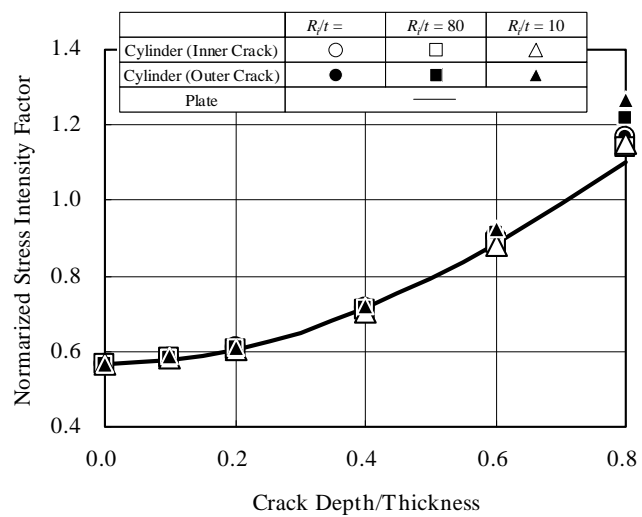

(c) Membrane Stress, Surface Point, $a / c=0.25$

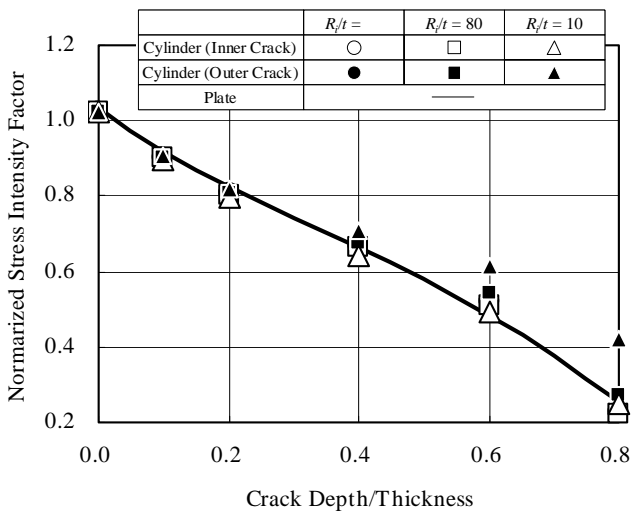

(b) Through-Wall Bending Stress, Deepest Point, $a / c=0.25$

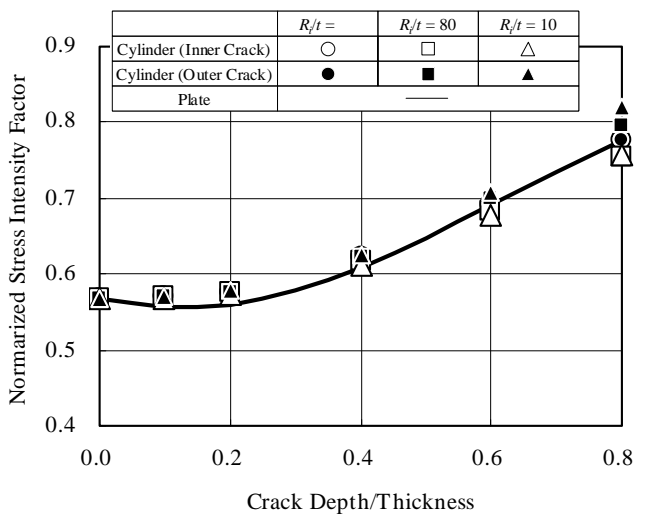

(d) Through-Wall Bending Stress, Surface Point, $a / c=0.25$

Fig. 7 Comparison of Stress Intensity Factors for Axially Semi-Elliptical Surface Cracked Cylinders and Semi-Elliptical Surface Cracked Plates

\subsection{Sample Case for Axially Infinite Surface Crack}

Figures 8(a) and (b) show examples of evaluation on the effect of radius-to-thickness ratio on the stress intensity factors for axially infinite surface cracked cylinders, where the notation of the axes is the same as Fig. 6. The normalized stress intensity factors for the equivalent single-edge cracked plates were also plotted on the figure. As the cylinder becomes thinner $\left(t / R_{i}\right.$ is smaller), the normalized stress intensity factors for inner and outer cracks increase, and they converge to the same value at $t / R_{i}=0$, which is quite close to the normalized stress intensity factor for the cracked plate, without regard to crack location (inner/outer surface) and applied loading (membrane/through-wall bending stress).

Figures 9(a) and (b) show the variation of the normalized stress intensity factors with crack depth. The normalized stress intensity factors for cracked cylinders coincide with those for the cracked plates for shallow cracks, while the normalized stress intensity factors for the thick cylinder $\left(R_{i} / t=10\right)$ with deeper cracks $($ alt $\varepsilon 0.6)$ are much smaller than those for cracked plates. Assuming that the allowable error of the stress intensity factor due to the replacement is tentatively $10 \%$, the upper bounds of allowable alt are $0.8,0.7$, and 0.4 for $R_{i} / t=400,80$, and 10 , respectively. However, it is proven that the replacement always gives conservative evaluation beyond the above limits. 


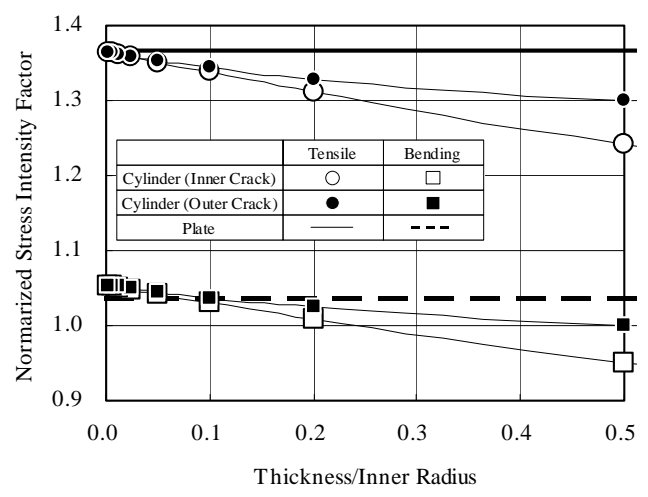

(a) $a / t=0.2$

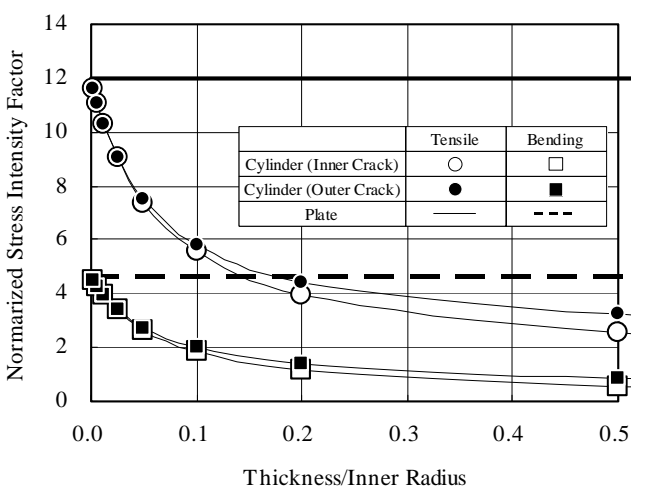

(b) $a / t=0.8$

Fig. 8 Effect of Radius-to-Thickness Ratio on Stress Intensity Factors for Axially Infinite Surface Cracked Cylinders

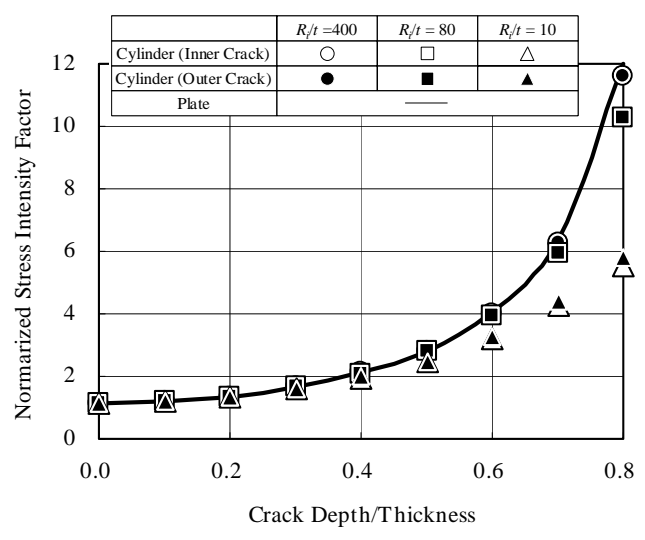

(a) Membrane Stress

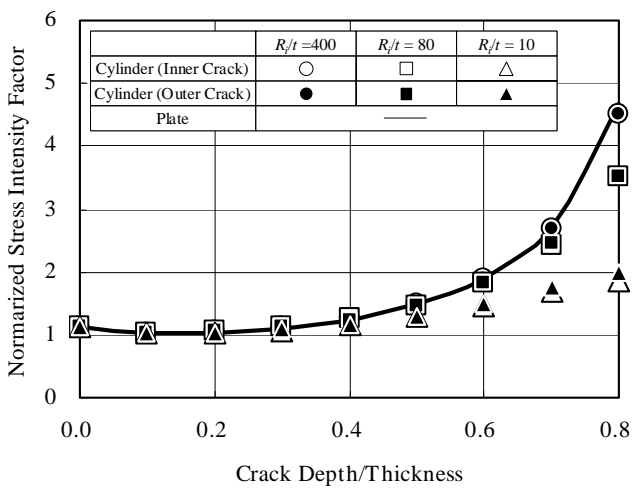

(b) Through-Wall Bending Stress

Fig. 9 Comparison of Stress Intensity Factors for Axially Infinite Surface Cracked Cylinders and Single-Edge Cracked Plates

\subsection{Other Types of Crack}

Similar examinations were also conducted for the replacement of circumferentially semi-elliptical surface cracked cylinders with semi-elliptical surface cracked plates, and for the replacement of circumferentially full-circumferential surface cracked cylinders with single-edge cracked plates. For the former case, the replacement exhibited the similar features with the case of axially semi-elliptical cracks, and was acceptable in the case of alt $\delta 0.6$ and $R_{i} / t \varepsilon 10$. For the latter case, the replacement again showed the similar features with the case of axially infinite cracks, however, the error due to the replacement was larger than the case of axial cracks especially for deeper cracks (a/t\& 0.5$)$. The replacement of thick cylinders with deeper cracks requires a special attention.

\section{CONCLUSIONS}

In this study, existing stress intensity factor solutions for cracked cylinders were reviewed and compared with each other. Generally good agreement was observed between available solutions for various types crack either axially or circumferentially expanded. Specific solutions were recommended for general use mainly from the viewpoints of the width of applicable geometry, applicable loading, and simplicity of evaluation. Approximate expressions were newly derived for axially through-wall cracked cylinder subjected to linear stress distribution and for circumferentially through-wall cracked cylinder subjected to trhrough-wall bending to facilitate the use of tabulated solutions. The adequacy of the replacement of cracked cylinders with cracked plates was then studied. 
The replacement of semi-elliptical surface cracked cylinders with the semi-elliptical surface cracked plates was found to be generally acceptable, while the replacement of cylinders with an infinite axial or full-circumference surface cracks with the single-edge cracked plates brings about large conservatism in the case of thick cylinders with deep cracks.

This study was performed under the sponsorship of the Ministry of Economy, Trade and Industry (METI) of Japan as a part of the project for developing new reactor technologies.

\section{REFERENCES}

[1] Chapuliot, S., (2000), Rapport CEA-R-5900.

[2] Andersson, P., Bergman, M., Brickstadt, B., Dahlberg, L., Nilsson, F., and Sattari-Far, I., (1996), SAQ/FoU-Report 96/08.

[3] Fett, T., Munz, D., and Neumann, J., (1990), Eng. Fract. Mech., Vol. 36, pp. 647.

[4] Raju, I. S., and Newman, J. C., (1962), ASME PVP, Vol. 58, pp. 37.

[5] American Petroleum Institute, (2000), API Recommended Practice 579.

[6] Shiratori, M., Miyoshi, T., Yu, Q., Terakado, T., and Matsumoto, T., (1999), ASME PVP, Vol. 385, pp. 299.

[7] Zahoor, A., (1985), Trans. ASME, J. Pres. Ves. Tech., Vol. 107, pp. 203.

[8] Drubay, B., Chapuliot, S., Lacire, M. H., and Marie, S., (2001) Rapport DM2S, SEMT/LISN/RT/01-025A.

[9] Wu, X. R., and Carlsson, A. J., (1991), "Weight Functions and Stress Intensity Factor Solutions," Pergamon Press, Oxford, UK.

[10] Erdogan, F., and Kibler, J. J., (1969), Int. J. Fract. Mech., Vol. 5, pp. 229.

[11] Folias, E. S., (1969), Int. J. Fract. Mech., Vol. 5, pp. 327.

[12] Green, D., and Knowles, J., (1994), Trans. ASME, J. Pres. Ves. Tech., Vol. 116, pp. 345.

[13] ASME Boiler and Pressure Vessel Code, Code Case N-513, (1997).

[14] France, C. C., Green, D., Sharples, J. K., and Chivers, T., (1997), ASME PVP, Vol. 350, pp. 143.

[15] Chapuliot, S., and Lacire, M. H., (1999), ASME PVP, Vol. 388, pp. 3.

[16] Bergman, M., (1995), Fatigue \& Fract. Eng. Mat. \& Struct., Vol. 18, pp. 1155.

[17] Lacire, M. H., Chapuliot, S., and Marie, S., (1999), ASME PVP, Vol. 388, pp. 13.

[18] Sattari-Far, I., (1992), Int. J. Fract., Vol. 53, pp. R9.

[19] Klecker, R., Brust, F., and Wilkowski, G. M., (1986), NUREG/CR-4572.

[20] Takahashi, Y., (2002), Int. J. Pres. Ves. Piping, Vol. 79, pp. 385.

[21] Newman, J. C. Jr., and Raju, I. S., (1984), NASA, Technical Memorandum, 85793.

[22] Tada, H., Paris, P. C., and Irwin, G. R., (2000), "The Stress Analysis of Cracks Handbook," ASME Press, New York, US. 